\title{
Primeiro registro de Fidicinoides picea (Walker, 1850) e Fidicinoides poulaini Boulard \& Martinelli, 1996 no Brasil
}

\author{
First report of Fidicinoides picea (Walker, 1850) and Fidicinoides poulaini Boulard \& Martinelli, 1996 \\ in Brazil
}

\author{
Rodrigo Souza Santos ${ }^{\mathrm{I}}$ Nilza Maria Martinelli ${ }^{\mathrm{II}}$
}

\begin{abstract}
As cigarras são importantes pragas de diversas culturas, entre plantas cultivadas e ornamentais. Estudos de taxonomia e morfologia de insetos são primordiais para pesquisas etológicas e para o emprego de métodos de controle. Ao estudar coleções entomológicas de cigarras, foi constatado o primeiro registro de ocorrência de Fidicinoides picea $e$ Fidicinoides poulaini (Hemiptera: Cicadidae) no Brasil, espécies coletadas nos Estados do Pará e de Manaus, respectivamente. Neste trabalho, são apresentadas as descrições morfológicas da cabeça, do tórax, do abdome e da asa.

Palavras-chave: Cicadoidea, Fidicinini, morfologia, taxonomia.
\end{abstract}

\section{ABSTRACT}

The cicadas are important pests of several cultivations, since cultivated until ornamental plants. Taxonomy and morphology studies of insects are primordial in researches of behavior and for the employment of control methods. Studying entomological collections of cicadas, it was verified the first report of occurrence of Fidicinoides picea and Fidicinoides poulaini (Hemiptera: Cicadidae) in Brazil, species collected in States of Pará and Manaus and are presented, morphologic descriptions of the head, thorax, abdomen and wing.

Key words: Cicadoidea, Fidicinini, morphology, taxonomy.
Na superfamília Cicadoidea, estão incluídas duas famílias Tettigarctidae e Cicadidae (MOULDS, 2005). Algumas espécies de Cicadidae são de importância econômica para o Brasil (METCALF, 1963), sendo consideradas pragas de plantas ornamentais e cultivadas, principalmente no cafeeiro (MARTINELLI \& ZUCCHI, 1989a; 1989b).

A tribo Fidicinini, pertencente à família Cicadidae, está representada por gêneros neotropicais, sendo caracterizada pela exposição pronunciada do orifício do órgão estridulatório (DISTANT, 1906). Os principais gêneros desta tribo que ocorrem no Brasil são Dorisiana, Proarna e Fidicina (METCALF, 1963), sendo que espécies de Fidicina e Dorisiana são de importância econômica, sugando a seiva das raízes e causando definhamento das plantas e perda de produtividade (MARTINELLI \& ZUCCHI, 1989a; 1989b; 1997). Ao revisarem esta tribo, BOULARD \& MARTINELLI (1996) descreveram o gênero Fidicinoides, caracterizado pelas placas láterometaescutelares curtas e estreitas, não atingindo os cimbacaliptos e não fechando completamente as câmaras cimbalares, separando, assim, do gênero Fidicina por apresentar o órgão estridulatório parcialmente exposto. Foram incluídas neste novo gênero as espécies Fidicinoides picea (Walker, 1850), Fidicinoides determinata (Walker, 1858), Fidicinoides

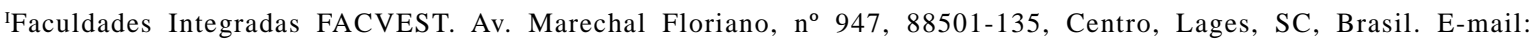
santos_rss@hotmail.com. Autor para correspondência.

"Departamento de Fitossanidade, Faculdade de Ciências Agrárias e Veterinárias (FCAV), Universidade Estadual Paulista (UNESP), Jaboticabal, SP, Brasil. 
pronoe (Walker, 1850) e Fidicinoides Iacteipennis (Distant, 1905), anteriormente pertencentes ao gênero Fidicina.

A fauna de cicadídeos no Brasil ainda é pouco conhecida, devido à pequena importância econômica que representavam no passado e à dificuldade de se estudar as coleções entomológicas dos mesmos, as quais se encontram dispersas em várias instituições brasileiras.

Nesse sentido, este trabalho tem como objetivos notificar o primeiro registro de ocorrência de F. picea e Fidicinoides poulaini Boulard \& Martinelli, 1996 no Brasil e apresentar características taxonômicas e morfológicas que possibilitem a identificação destas espécies nativas.

O estudo incluiu exemplares de cigarras provenientes de seis instituições brasileiras (Fundação Oswaldo Cruz - Rio de Janeiro (RJ); Instituto Nacional de Pesquisas da Amazônia/INPA - Amazonas (AM); Faculdade de Ciências Agronômicas (FCA/UNESP), Botucatu - São Paulo (SP); Museu Nacional do Rio de Janeiro/MNRJ - Rio de Janeiro (RJ); Instituto Florestal de São Paulo - SP e Universidade Federal do Mato Grosso (UFMT) - Mato Grosso (MT)) e uma internacional (Musèum National d'Histoire Naturelle/ MNHN-Paris).

As espécies foram comparadas com os respectivos holótipos e parátipos, depositados na Coleção Entomológica de Cicadídeos, do Departamento de Fitossanidade, da Faculdade de Ciências Agrárias e Veterinárias (FCAV/UNESP), localizada em Jaboticabal, SP. Os exemplares estudados foram colocados em câmara úmida e, posteriormente, foram efetuadas montagens com as asas distendidas em posição oblíqua ao corpo, com o objetivo de serem obtidos os exemplares em melhores condições para observação.

Para o estudo da genitália masculina, separou-se o abdome com auxílio de pinça, submetendo-o ao aquecimento em banho-maria, em solução de hidróxido de potássio ( $\mathrm{KOH}$ a 5\%), durante aproximadamente 30 minutos, verificando o processo de clarificação da peça. Posteriormente, esta foi lavada em água destilada até a eliminação de todo o material do interior do corpo, para posterior exame sob microscópio estereoscópico (lentes Carl Zeiss Jena ${ }^{\circledR}$ ). As genitálias foram acondicionas em tubos plásticos tipo “eppendorf” (4cm de altura), devidamente etiquetados, contendo glicerina (60\%), e depois foram comparadas com as descrições efetuadas por BOULARD \& MARTINELLI(1996).

As figuras para a caracterização morfológica das espécies foram ilustradas com auxílio de câmara clara acoplada ao microscópio estereoscópico. Foram ilustradas: cabeça e tórax (vista dorsal), abdome (vista ventral), asa anterior direita e último urosternito do macho, sendo que tais ilustrações não constam no trabalho de BOULARD \& MARTINELLI (1996). Foram omitidas as ilustrações da genitália masculina, do opérculo e do fêmur anterior direito, as quais foram apresentadas por BOULARD \& MARTINELLI (1996). As medidas foram tomadas com auxílio de uma régua milimetrada.

Fidicinoides picea (Walker, 1850)

Principais sinonímias: Fidicina picea Walker, 1850; Fidicina pertinax Stål, 1864; Fidicina determinata Distant, 1881, Metcalf, 1963; Fidicina picea Wolda \& Ramos, 1992.

Cabeça (Figura 1A): ocelos laterais e mediano muito próximos entre si, situados em uma longa mancha escura, de contorno irregular, estendendo-se até a base das antenas, mas não atingindo o vértice, distância entre os ocelos laterais cerca de duas vezes maior que a distância que os separam dos olhos correspondentes, manchas suboculares não-unidas, rostro longo, com seu ápice estendendo-se entre o segundo e o terceiro par de pernas.

Tórax (Figura 1A): pronoto duas vezes mais longo que a cabeça e o pardacento, lobos suprahumerais pouco desenvolvidos, mesonoto pardacento e verde, portando quatro manchas pretas e simétricas (duas externas longas e estreitas, duas internas igualmente estreitas e em formato de vírgula, uma mancha estreita e duas pequenas manchas circulares, pretas, acima da elevação cruciforme) (Figura 1A).

Abdome (Figura 1B): pardacento, um pouco mais longo que a distância compreendida do vértice aos ápices posteriores da elevação cruciforme, cimbacaliptos rudimentares, último urosternito do macho (Figura 1D), cerca de duas vezes mais largo que longo, com leve depressão na margem anterior, margem lateral oblíqua e levemente côncava na sua metade, margem posterior côncava e arredondada, com ápices afastados entre si.

Asa anterior (Figura 1C): hialinas; com célula basal opaca, de coloração parda-escura, costa pardacenta igualando com a metade da asa, nervuras marrons.

Material examinado: BRASIL. Pará: Cachimbo. Fundação Oswaldo Cruz. 20.VII.1955. (L. Travassos, S. Oliveira \& Pearson), $1 \hat{\delta}$; ibidem, 21.IX.1955. (L. Travassos \& S. Oliveira), 1 ; ibidem, 09.X.1956. (L. Travassos, S. Oliveira \& Adão), 10 .

Comentário: Espécie de porte médio, próxima de $\boldsymbol{F}$. pronoe, diferindo desta por apresentar as manchas externas do mesonoto não atingindo a elevação cruciforme. Anteriormente citada no gênero Fidicina, trata-se da primeira ocorrência desta espécie no Brasil, no Estado do Pará, sendo relatada também no México, na Venezuela, na Colômbia e no Equador (BOULARD \& MARTINELLI, 1996). 


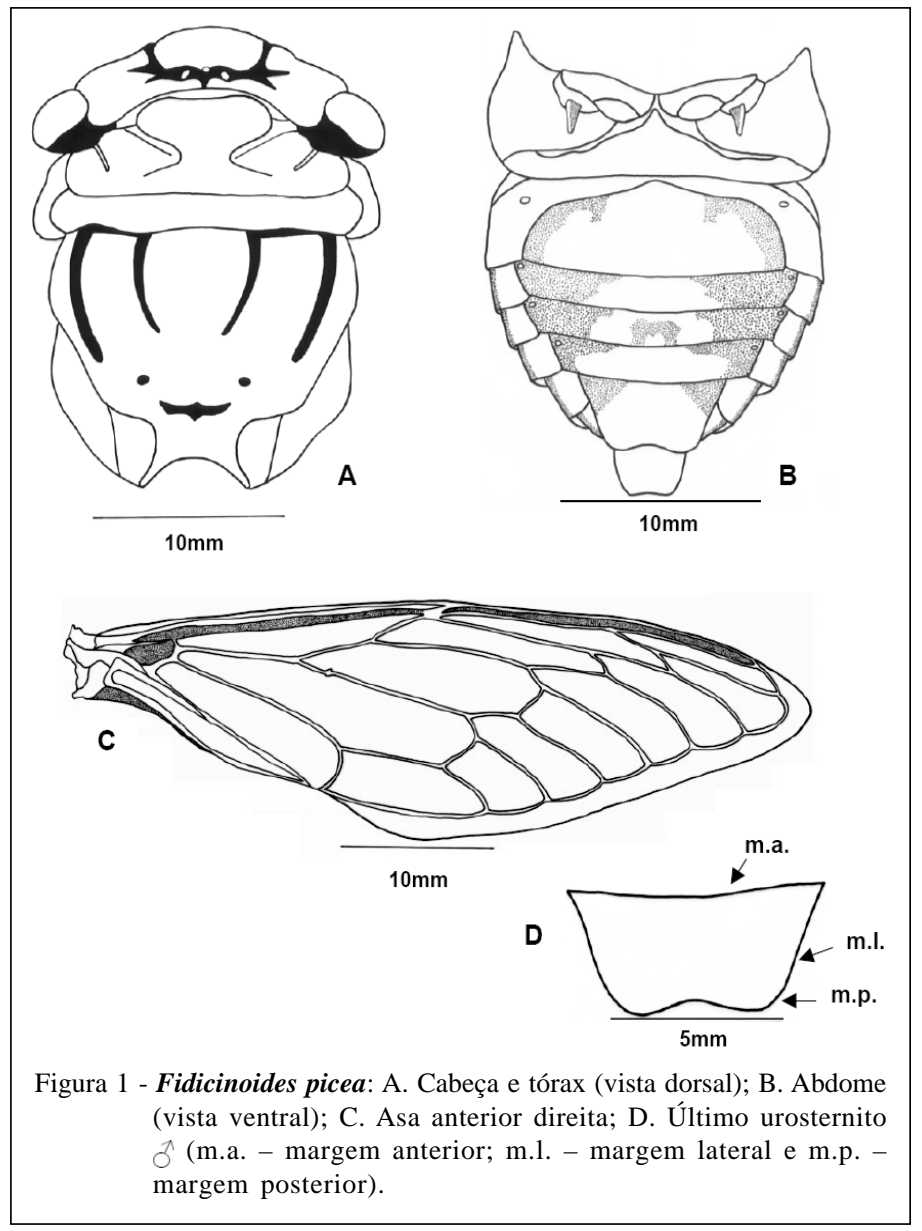

Fidicinoides poulaini Boulard \& Martinelli, 1996. Cabeça (Figura 2A): ocelos laterais e mediano situados em uma mancha escura, de contorno irregular, não estendendo até a base das antenas e do vértice, distância compreendida entre os ocelos laterais cerca de duas vezes e meia maior que a distância que os separam dos olhos correspondentes, uma mancha estreita e uma mancha escura entre a cabeça e a área interna do pronoto, manchas suboculares não-unidas, rostro longo, com ápice alcançando a metade das coxas posteriores.

Tórax (Figura 2A): pronoto mais longo que a cabeça; lobos supra-humerais pouco desenvolvidos, mesonoto esverdeado, portando quatro manchas (duas manchas externas escuras próximas ao pronoto, área central gradualmente mais clara, escurecendo novamente nos ápices posteriores, duas manchas internas curtas, escuras e em formato de vírgula, uma mancha em forma de losango e duas pequenas manchas circulares, pretas, acima da elevação cruciforme) (Figura 2A).
Abdome (Figura 2B): mais curto que a distância compreendida do vértice aos ápices posteriores da elevação cruciforme, base larga se estreitando em direção ao último segmento abdominal, cimbacaliptos pretos, último urosternito do macho (Figura 2D) cerca de uma vez e meio mais largo que longo, margem anterior com pequena depressão central, margem lateral oblíqua e margem posterior com invaginação acentuada, ápices próximos entre si.

Asa anterior (Figura 2C): hialinas, esfumaçadas de pardo claro na área apical, com célula basal quase totalmente opaca de ocre esverdeado, costa longa e verde, nervuras ocres, tornando-se pardas na área apical, na área apical octoloculada.

Material Examinado: PERU. Loreto. Requema. 16.X.1991. Muséum National d'Histoire Naturelle, MNHN/Paris. (Poulain, S.), 1 parátipo BRASIL. Pará: Cachimbo. Fundação Oswaldo Cruz. 09.X.1956. (Travassos, Oliveira \& Adão), 110 ; ibidem, 21.IX.1955. (L. Travassos \& S. Oliveira), 10 ; Amazonas: Manaus. MN/RJ. X.1955. (Elias \& Roppa), 1. 


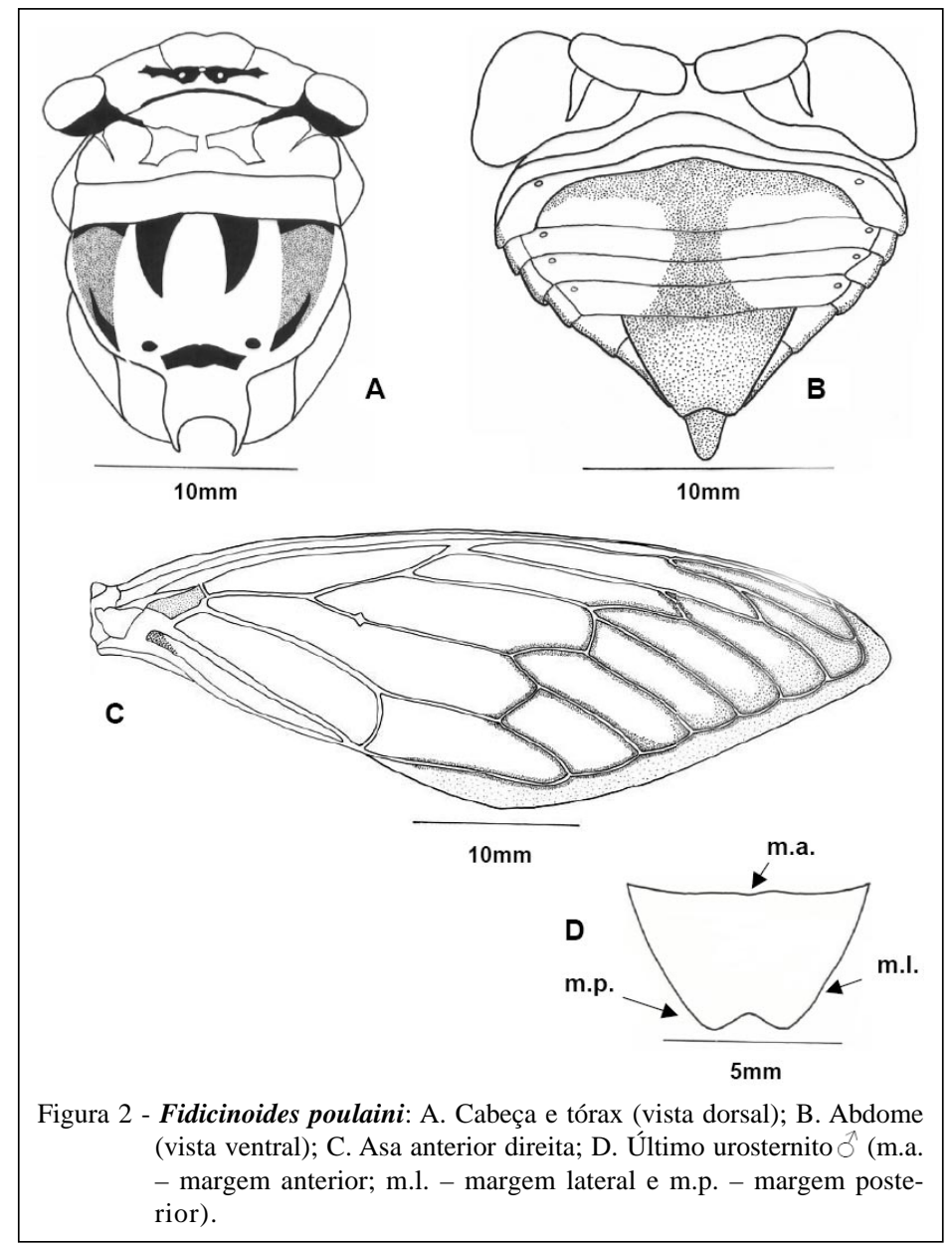

Comentário: Espécie de porte médio, próxima de Fidicinoides duckensis (Boulard \& Martinelli, 1996), diferindo desta por apresentar o último urosternito do macho com uma pequena depressão na sua margem anterior e margem lateral levemente convexa. Trata-se da primeira ocorrência desta espécie no Brasil, nos Estados do Pará e de Manaus, sendo anteriormente relatada no Peru e no Equador (BOULARD \& MARTINELLI, 1996).

Em vista do exposto e das características demonstradas neste trabalho, foi possível identificar a primeira ocorrência das espécies $\boldsymbol{F}$. picea e $\boldsymbol{F}$. poulaini nos Estados do Pará e de Manaus, respectivamente, contribuindo para o conhecimento e a identificação da fauna de cicadídeos do Brasil.

\section{REFERÊNCIAS}

BOULARD, M.; MARTINELLI, N.M. Révision des fidicinini; noveau statut de la tribu, espèces connues et nouvelles espèces (Cicadomorpha, Cicadidae, Cicadinae). EPHE, Biologie et Evolution des Insectes, Paris, v.9, p.11-81, 1996.
DISTANT, W.L. A synomyc catalogue of Homoptera, Part I, Cicadidae. Londres: British Museum, 1906. 207p.

MARTINELLI, N.M.; ZUCCHI, R.A. Cigarras associadas ao cafeeiro. II. Gênero Fidicina Amyot \& Serville, 1843 (Homoptera, Cicadidae, Cicadinae). Anais da Sociedade Entomológica do Brasil, Londrina, v.18, n.1, p.5-12, 1989a.

MARTINELLI, N.M.; ZUCCHI, R.A. Cigarras associadas ao cafeeiro. III. Gênero Dorisiana Metcalf, 1952 (Homoptera, Cicadidae, Cicadinae). Anais da Sociedade Entomológica do Brasil, Londrina, v.18, n.1, p.5-12 (supl.), 1989b.

MARTINELLI, N.M.; ZUCCHI, R.A. Primeiros registros de plantas hospedeiras de Fidicina mannifera, Quesada gigas e Dorisiana drewseni (Hemiptera: Cicadidae). Revista de Agricultura, Piraciba, v.72, n.3, p.271-281, 1997.

METCALF, Z.P. General Catalogue of the Homoptera, Cicadoidea, Parts 1-2. Raleigh: Waverly, 1963. v.8, p.1919.

MOULDS, M.S. An appraisal of the higher classification of cicadas (Hemiptera: Cicadoidea) with special reference to the Australian fauna. Records of the Australian Museum, Sydney, v.57, n.3, p.375-446, 2005. 